\title{
Exercise performance and quality of life following surgical repair of anomalous aortic origin of a coronary artery in the pediatric population
}

\author{
J. A. Brothers, MD, ${ }^{a}$ M. G. McBride, PhD, ${ }^{a}$ B. S. Marino, MD, MPP, MSCE, ${ }^{\mathrm{b}}$ R. S. Tomlinson, BSE, ${ }^{\mathrm{b}}$ M. A. Seliem, MD, \\ M. H. Pampaloni, MD, PhD, ${ }^{c}$ J. W. Gaynor, MD, ${ }^{d}$ T. L. Spray, MD, ${ }^{d}$ and S. M. Paridon, MD ${ }^{a}$
}

\begin{abstract}
Objective: We sought to evaluate exercise performance and quality of life in children after surgical repair of anomalous aortic origin of a coronary artery with an interarterial course.

Methods: Patients who had surgery from October 2001 to January 2007 were eligible for inclusion. Exercise performance and quality of life were prospectively assessed by maximal exercise tests and age-appropriate questionnaires, respectively. We used $t$ tests to compare pre- and postoperative exercise data and quality-of-life scores to published normative data. We performed linear regression analyses to assess associations between demographic, anatomic, and exercise variables and quality-of-life score.
\end{abstract}

Results: Of $25 / 27$ patients, $64 \%$ were boys, $68 \%$ had anomalous right coronary, $32 \%$ were asymptomatic. Average age at surgery was $10.8( \pm 4.1)$ years; median follow-up was 14.5 ( 2 to 48$)$ months. Postoperative percent-predicted exercise values were: peak heart rate $97( \pm 6)$, working capacity $91( \pm 15)$, maximal oxygen consumption $82( \pm 16)$. In those who had preoperative exercise testing $(n=11)$, resting and maximal heart rates decreased significantly without significant change in exercise performance. Average child quality of life was $85 / 100( \pm 13)$ and parent-proxy $88( \pm 11)$ compared with normal scores of $83( \pm 15)$ and $88( \pm 12)$, respectively.

Conclusion: There is mild chronotropic impairment in children and adolescents following anomalous coronary artery repair without a decline in exercise performance. This does not appear to impair their overall quality of life. Because long-term effects on heart rate, exercise performance, and quality of life are unknown, serial exercise tests should be included as routine care of these patients.

Anomalous aortic origin of a coronary artery that courses between the 2 great arteries (AAOCA) is a rare anomaly where the left main coronary artery arises from the right sinus of Valsalva (ALCA) or the right arises from the left sinus (ARCA). AAOCA is associated with increased risk of sudden cardiac death, notably among otherwise healthy athletic children and young adults. ${ }^{1-8}$ The most common presenting complaints are cardiovascular in origin and include chest pain, dizziness, or syncope during or just after exertion. ${ }^{4,9,10}$ Although most physicians would agree that surgery is indicated if a patient presents with signs and/or symptoms of myocardial ischemia, significant controversy exists regarding treatment of asymptomatic patients, especially those with ARCA, where the sudden death risk appears to be lower. Some physicians advocate either no intervention or exercise restriction, whereas others recommend surgical repair.

\footnotetext{
From the Division of Cardiology, ${ }^{\mathrm{a}}$ Department of Anesthesiology and Critical Care, Department of Radiology, ${ }^{\mathrm{c}}$ Division of Cardiothoracic Surgery, ${ }^{\mathrm{d}}$ The Children's Hospital of Philadelphia and the University of Pennsylvania, Philadelphia, Penn. Dr. Brothers was supported by an NIH Institutional National Research Service Award Training Grant (T32HL007915).

Received for publication April 28, 2008; revisions received July 8, 2008; accepted for publication Aug 4, 2008.

Address for reprints: Julie A. Brothers, MD, The Children's Hospital of Philadelphia, 34th and Civic Center Blvd, Division of Cardiology, Main Building, 8NW Room 72, Philadelphia, PA 19104 (E-mail: brothersj@email.chop.edu).

J Thorac Cardiovasc Surg 2009;137:380-4

$0022-5223 / \$ 36.00$

Copyright (c) 2009 by The American Association for Thoracic Surgery doi:10.1016/j.jtcvs.2008.08.008
}

In patients who have surgery, exercise is usually restricted postoperatively until they have had, at minimum, a formal exercise stress test. ${ }^{11}$ Exercise limitations may have a significant impact on children's quality of life (QOL) not only by affecting their physical capabilities but also by influencing their cognitive and psychosocial health. The effects of changes in postoperative exercise performance, defined as maximal aerobic capacity and physical working capacity, have not previously been reported. The purpose of this study was to prospectively evaluate exercise performance and QOL in children with AAOCA after surgical repair.

\section{MATERIALS AND METHODS \\ Subjects}

After approval from the Institutional Review Board at The Children's Hospital of Philadelphia, patients were identified by review of our Cardiothoracic Surgical database. Inclusion criteria included all patients diagnosed with AAOCA who had surgical repair at our institution between October 2001 and January 2007. Patients with anomalous coronary from the pulmonary artery, other coronary artery anomalies (eg, coronary-cameral fistula, coronary aneurysms, myocardial bridging), or other significant structural heart disease were excluded from the study.

After obtaining parental consent and child assent, each patient's medical record was reviewed for presenting signs and symptoms and preoperative exercise stress test (EST). We defined cardiovascular symptoms as chest pain, presyncope, or syncope occurring with or just after exertion; cardiovascular signs were defined as ventricular arrhythmia, exercise-induced ischemia, myocardial infarction, or aborted sudden death. Data regarding any postoperative EST performed prior to our evaluation were also collected. 


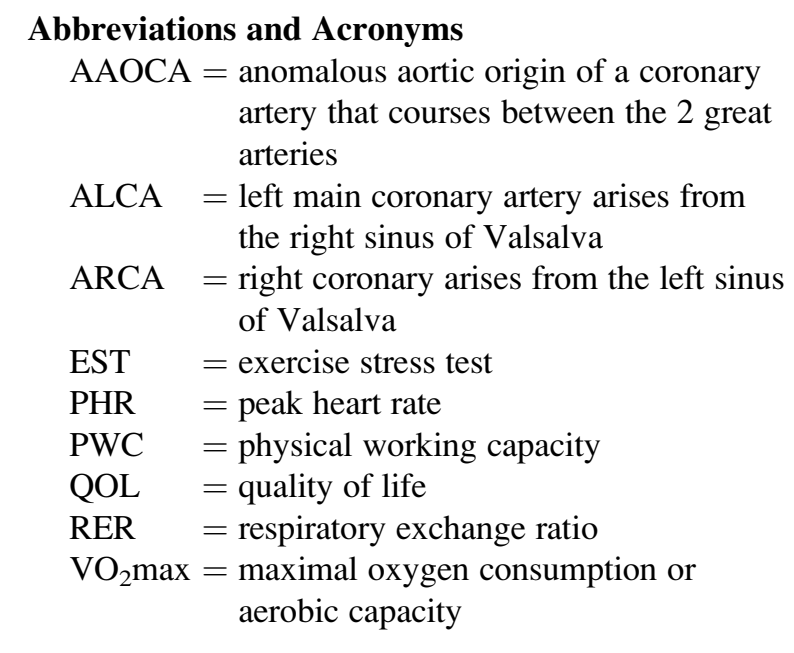

Enrolled patients had prospective exercise testing and completed QOL questionnaires as described below.

\section{Exercise Protocol, Cardiac Monitoring, and Metabolic Measurements}

Exercise protocol. Patients exercised to maximal ability using an electronically braked cycle ergometer (SensorMedics, Yorba Linda, CA). They pedaled for 3 minutes at an unloaded state followed by a ramp increase in work rate to maximal exercise. Four subjects who did not meet the height requirements $(>130 \mathrm{~cm})$ for cycle ergometry exercised on a treadmill (Marquette Series 2000, Milwaukee, WI) using a 1-minute incremental protocol. Four subjects tested at an outside hospital exercised on a treadmill using a similar protocol.

Cardiac monitoring. A 12-lead electrocardiogram (Marquette Case-8000) was obtained at rest in the supine, sitting, and standing positions; during each minute of exercise; and during the first 10 minutes of recovery. Cardiac rhythm was monitored continuously throughout the study. Blood pressure was measured by auscultation at rest and every 3 minutes during exercise and recovery.

Metabolic measurements. Metabolic data were obtained throughout the study and for the first 2 minutes of recovery on a breath-by-breath basis using a metabolic cart (SensorMedics V29, Yorba Linda, CA). Measured variables included: maximal oxygen consumption or aerobic capacity $\left(\mathrm{VO}_{2} \mathrm{max}\right)$, physical working capacity (PWC), peak heart rate (PHR), and respiratory exchange ratio (RER). Exercise performance was defined by $\mathrm{VO}_{2} \max$ and PWC. The ventilatory anaerobic threshold was measured by the V-slope method. ${ }^{12}$ Data were expressed as percent-predicted values from age- and gender-matched normative data used in our laboratory. ${ }^{13}$ Values were indexed for ideal body weight in those patients whose weight exceeded $110 \%$ of ideal body weight at the time of exercise test. ${ }^{14}$ A maximal EST was defined as attaining a ratio of carbon dioxide produced to oxygen consumed at maximal exercise (i.e., RER) of $\geq 1.10$ and/or attaining a maximum heart rate of $\geq 85 \%$ of predicted.

\section{QOL Questionnaires}

To assess health-related QOL, we utilized the Peds QL Pediatric Quality of Life Inventory, Version 4.0. ${ }^{15-18}$ There were separate questionnaires for patient (self-report) and parent (parent-proxy report). These consisted of 23 questions regarding core dimensions of health: physical, emotional, social, and school functioning within the past month. The questionnaires were divided by age into young-child (5 to 7 years), child ( 8 to 12 years), and teen
TABLE 1. Presenting signs and symptoms $(n=25)$

\begin{tabular}{lll}
\hline \multicolumn{1}{c}{ Symptom } & $\mathbf{n}(\%)$ & \multicolumn{1}{c}{ Coronary anomaly } \\
\hline Cardiovascular symptoms & & \\
$\quad$ Chest pain, exercise & $6(24)$ & ARCA \\
Presyncope, exercise & $1(4)$ & ALCA \\
Syncope, exercise & $3(12)$ & ALCA $(\mathrm{n}=2)$, ARCA $(\mathrm{n}=1)$ \\
$\begin{array}{l}\text { Aborted sudden death, } \\
\quad \text { exercise }\end{array}$ & $1(4)$ & ALCA \\
$\quad \begin{array}{l}\text { Palpitations/PVCs } \\
\text { Other symptoms }\end{array}$ & $1(4)$ & ALCA \\
$\begin{array}{l}\text { Chest pain, rest } \\
\text { Presyncope, rest }\end{array}$ & $3(12)$ & ARCA $(\mathrm{n}=2)$, ALCA $(\mathrm{n}=1)$ \\
$\quad$ Syncope, rest & $1(4)$ & ARCA \\
Asymptomatic & ARCA \\
$\quad \begin{array}{l}\text { Murmur evaluation } \\
\text { F/U Kawasaki disease }\end{array}$ & $6(24)$ & ARCA $(\mathrm{n}=4)$, ALCA $(\mathrm{n}=2)$ \\
$\quad$ Family history & $1(4)$ & ARCA \\
\hline
\end{tabular}

$A L C A$, Anomalous left main coronary artery; $A R C A$, anomalous right coronary artery; $F / U$, follow-up; $P V C s$, premature ventricular contractions.

(13 to 18 years) groups. A total score, as well as physical and psychosocial health summary scores, was generated. Scores ranged from 0 (worst functioning) to 100 (best functioning).

\section{Statistical Analysis}

Baseline clinical characteristics were described. Measured EST metabolic variables were summarized as mean \pm standard deviation and reported as percent-predicted values compared to age- and sex-matched normal values. ${ }^{18}$ Pre- and postoperative EST data were compared by paired $t$ tests. We compared average QOL questionnaire scores to normal values from healthy children using 2 -sample $t$ test with unequal variances. ${ }^{15}$ Linear regression analyses were performed to assess the association between demographic, anatomic, and exercise variables and QOL score. All tests of significance were 2-tailed.

\section{RESULTS}

\section{Baseline Demographics}

Twenty-seven consecutive patients met criteria for study inclusion. Twenty-five $(95 \%)$ consented to participate; the 2 not included were either lost to follow-up or declined participation. Of the 25 patients, $16(64 \%)$ were boys and $17(68 \%)$ had ARCA. The average age at surgery was $10.8( \pm 4.1)$ years, and the median time to follow-up was 14.5 (2 to 48) months. Presenting signs and symptoms and the associated coronary anomaly are shown in Table 1 . Seventeen patients had symptoms at diagnosis; we classified 12 as cardiovascular in origin (ie, occurring with exercise or an arrhythmia).

\section{Preoperative Exercise Tests}

Preoperative EST was performed in $17(68 \%)$ patients, 11 of whom had complete metabolic data. All performed a maximal EST based on attaining at least $85 \%$ of predicted maximal heart rate and/or RER $>1.10$. Although we routinely obtained preoperative EST, 13 patients had already had surgery at study initiation. Seven of these $13 \mathrm{had}$ a preoperative EST. Ten of 12 who had surgery after study onset had preoperative EST. 
TABLE 2. Postoperative exercise test results $(n=21)$

\begin{tabular}{lcc}
\hline \multicolumn{1}{c}{ Variable } & Value $( \pm$ SD) & $\%$ Predicted $( \pm$ SD $)$ \\
\hline $\mathrm{VO}_{2} \max (\mathrm{mL} / \mathrm{kg} / \mathrm{min})$ & $35.3(7.3)$ & $81.8(15.7)$ \\
$\mathrm{AT}(\mathrm{mL} / \mathrm{kg} / \mathrm{min})$ & $19.5(4.2)$ & $79.1(16.3)$ \\
$\mathrm{PWC}(\mathrm{W})$ & $171.5(62.6)$ & $91.2(14.5)$ \\
$\mathrm{PHR}(\mathrm{bpm})$ & $190.8(7.7)$ & $97.1(5.6)$ \\
$\mathrm{RER}$ & $1.21(0.15)$ & $\mathrm{n} / \mathrm{a}$ \\
\hline
\end{tabular}

$A T$, Anaerobic threshold; $B P M$, beats per minute; $P H R$, peak heart rate; $P W C$, physical working capacity; $R E R$, respiratory exchange ratio; $S D$, standard deviation; $\mathrm{VO}_{2} \max$, maximal oxygen consumption; $W$, watts. ${ }^{*} \mathrm{n}=17$ who exercised on bicycle.

\section{Postoperative Exercise Tests}

All but 1 patient performed a maximal EST as defined above, and all were asymptomatic during testing. Indication to stop was patient fatigue as per standard protocol in our laboratory. Complete metabolic data were available on 21 patients as shown in Table 2. Four patients had EST at outside institutions that did not include metabolic assessments. Our patients had low-normal aerobic capacity with normal physical working capacity postoperatively. A plot of postoperative individual percent-predicted $\mathrm{VO}_{2}$ max efforts is illustrated in Figure 1. Table 3 presents data on the 11 patients who had complete metabolic data from pre- and postoperative exercise testing. As expected, patients were significantly taller and heavier postoperatively. Although resting and maximal heart rates were significantly lower $(P=.05$ and .006 , respectively), there was no significant difference in percent-predicted $\mathrm{VO}_{2}$ max or PWC postoperatively compared with preoperatively. In the 9 patients who had evidence of ischemia with exercise, as noted in a previous article of this same cohort, ${ }^{9}$ there was no significant difference in heart rate, $\mathrm{VO}_{2} \mathrm{max}$, or $\mathrm{PWC}$ when compared with those patients without evidence of ischemia.

\section{QOL Questionnaires}

Age-appropriate QOL questionnaires were administered to each patient and parent as described above. As shown

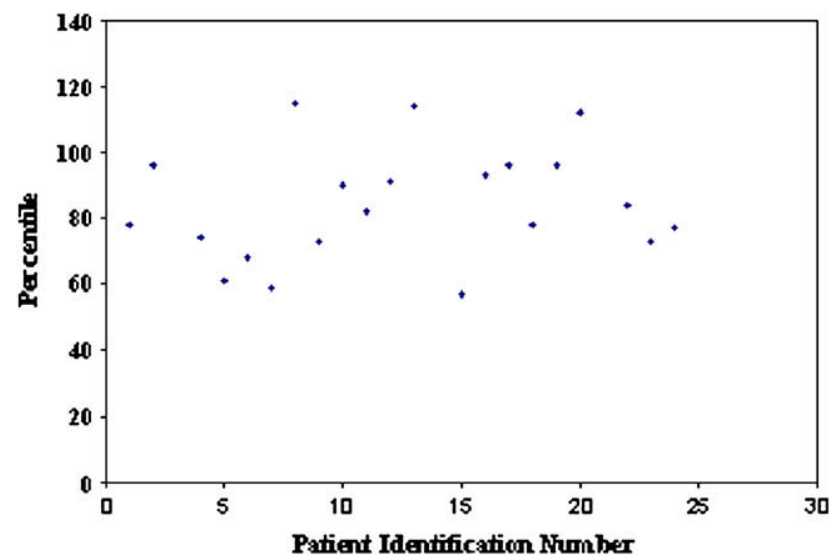

FIGURE 1. Postoperative percent-predicted maximal oxygen consumption.
TABLE 3. Preoperative and postoperative exercise test results $(\mathbf{n}=\mathbf{1 1})$

\begin{tabular}{|c|c|c|c|}
\hline Variable & Preoperative & Postoperative & $P$ value \\
\hline Age $(y)$ & $11.3( \pm 3.2)$ & $13.0( \pm 3.6)$ & .001 \\
\hline Gender ( $\%$ male $)$ & $72.7 \%$ & $\mathrm{n} / \mathrm{a}$ & $\mathrm{n} / \mathrm{a}$ \\
\hline Height $(\mathrm{cm})$ & $152.9( \pm 20.1)$ & $160.2( \pm 17.9)$ & .01 \\
\hline Weight $(\mathrm{kg})$ & $48.9( \pm 18.4)$ & $56.9( \pm 18.7)$ & .004 \\
\hline Resting HR (bpm) & $79.8( \pm 7.3)$ & $70.4( \pm 13.8)$ & .05 \\
\hline Maximal HR (bpm) & $197.1( \pm 5.0)$ & $190.0( \pm 9.4)$ & .006 \\
\hline Maximal HR ( $\%$ predicted) & $99.4( \pm 4.8)$ & $96.8( \pm 6.5)$ & .08 \\
\hline Resting SBP (mm Hg) & $115.0( \pm 14.9)$ & $108.4( \pm 11.1)$ & .13 \\
\hline Maximal SBP $(\mathrm{mm} \mathrm{Hg})$ & $153.9( \pm 32.0)$ & $155.4( \pm 36.9)$ & .92 \\
\hline Maximal SBP (\% predicted) & $99.5( \pm 13.3)$ & $95.2( \pm 16.1)$ & .24 \\
\hline $\mathrm{VO}_{2} \max \left(\mathrm{mL} \cdot \mathrm{kg}^{-1} \cdot \min ^{-1}\right)$ & $37.4( \pm 6.1)$ & $36.8( \pm 6.3)$ & .72 \\
\hline $\mathrm{VO}_{2} \max (\%$ predicted $)$ & $89.0( \pm 11.2)$ & $89.2( \pm 11.4)$ & .97 \\
\hline AT $(\mathrm{mL} / \mathrm{kg} / \mathrm{min})$ & $21.4( \pm 4.3)$ & $20.9( \pm 3.9)$ & 0.72 \\
\hline AT $(\%$ predicted $)$ & $86.3( \pm 17.6)$ & $85.1( \pm 15)$ & .85 \\
\hline PWC (W) & $151.2( \pm 59.4)$ & $178.8( \pm 68.1)$ & .03 \\
\hline PWC ( $\%$ predicted $)$ & $102.0( \pm 11.9)$ & $96.1( \pm 11.5)$ & .08 \\
\hline RER & $1.19( \pm 0.09)$ & $1.19( \pm 0.08)$ & 0.97 \\
\hline
\end{tabular}

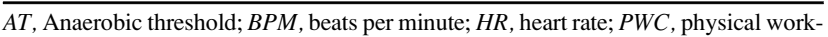
ing capacity; $R E R$, respiratory exchange ratio; $S B P$, systolic blood pressure; $V_{2}$ max, maximal oxygen consumption; $W$, watts.

in Table 4, our patients had a normal QOL following surgical repair when compared with healthy children and adolescents. There were no associations between age at surgery, length of follow-up, aerobic capacity, coronary anatomy, and QOL score.

\section{DISCUSSION}

Our study found that patients after AAOCA repair had low-normal aerobic capacity and normal physical working capacity that were not adversely affected by surgery. Although impairment in aerobic capacity before surgery may represent physician- and parent-imposed exercise restrictions, the lack of improvement postoperatively is unclear. There were a few patients tested early postoperatively who may have still been exercise-restricted by their physician, but the majority of patients were tested after receiving exercise clearance. We can speculate that children postoperatively may still be limited by their parents or by

TABLE 4. Quality-of-life results

\begin{tabular}{lccc}
\hline Health variable & Score $( \pm$ SD) & Normal value $( \pm$ SD) & $P$ value \\
\hline Patient & & & \\
$\quad$ Physical & $89.0(12.9)$ & $84.4(17.3)$ & 0.19 \\
$\quad$ Psychosocial & $82.4(14.9)$ & $82.4(15.5)$ & 1.0 \\
$\quad$ Total & $84.9(12.6)$ & $83.0(14.8)$ & 0.53 \\
Parent-proxy & & & \\
$\quad$ Physical & $93.0(10.0)$ & $89.3(16.4)$ & 0.26 \\
$\quad$ Psychosocial & $84.9(13.7)$ & $86.6(12.8)$ & 0.52 \\
$\quad$ Total & $87.6(11.2)$ & $87.6(12.3)$ & 1.0 \\
\hline
\end{tabular}

Scoring range from 0 to $100: 0=$ worst functioning, $100=$ best functioning. $S D$, Standard deviation. 
themselves, notably in those activities that require submaximal or maximal efforts.

Importantly, these children and adolescents had lower resting and maximal heart rates postoperatively despite exercising to the same degree before and after surgery. The reason for this finding is unclear. It is possible that this indicates a decrease in sympathetic tone, which could potentially be a consequence of manipulating the aortic root as part of the surgical repair. Resting heart rate should be less impacted than the maximal heart rate because resting sympathetic tone should contribute less to the regulation of the resting heart rate. Further, the decrease in heart rate cannot be explained by the children getting older, as the average follow-up time was in months, not years. Despite this, patients were able to compensate with either an increased stroke volume and/or an increase in muscle oxygen extraction, which enabled them to maintain their exercise performance. We do not know if this chronotropic impairment will continue to worsen over the next several years. For this reason, these children should be followed over time to continue evaluating for increasing chronotropic impairment that may, at some point, start to cause a decrease in exercise performance.

When making the decision to recommend repair of AAOCA, especially in patients who are asymptomatic, one also needs to consider that most of these children are otherwise healthy and have been leading normal lives. Even if the surgery proceeds well, it can potentially have a major impact on their lives. This impact has never been evaluated previously in the AAOCA population. Although we did not have evaluations prior to surgery, we found that children and their parents after AAOCA repair reported a normal QOL score. QOL has been evaluated in children after heart transplantation. These children do not have a normal QOL after surgery, largely due to decreased exercise tolerance, medication burden, number of medical visits required, and concern for infection or graft vasculopathy, all of which can lead to death. ${ }^{19,20}$ In the AAOCA population, where surgery was performed specifically because of the increased risk of a sudden catastrophic event, it appears that neither the patients nor their parents perceive a continued risk of sudden death postoperatively that is discernible by QOL assessment. Furthermore, neither the child's nor the parent's assessment of QOL correlated with the child's exercise capabilities.

\section{Limitations}

First, this was a relatively small study population; however, our institution has the largest cohort of pediatric patients who had AAOCA surgery and had exercise performance and QOL assessed. Second, the study was initiated after surgical repair. Because of this, preoperative exercise tests with complete metabolic data were not available on several patients; our cohort of patients who had both tests may not be representative of the larger patient population. QOL data were also missing preoperatively. Although we found that QOL was normal after surgery, we do not know if it was normal or abnormal prior to surgery. Because of this, we can only speculate that surgery improved QOL by removing exercise restrictions and diminishing parent and patient fear of sudden death.

\section{CONCLUSIONS}

There is evidence of mild chronotropic impairment in children and adolescents following AAOCA surgery. This was not associated with a decrease in exercise performance or diminished QOL. However, long-term effects of AAOCA surgery are unknown. If future evaluations demonstrate continued chronotropic impairment or a decline in exercise performance or QOL, then the threshold for surgical repair in asymptomatic patients should be increased. For these reasons, it is important to follow these patients with serial exercise tests and QOL assessments to evaluate if there are changes in chronotropy, exercise performance, and QOL over the next several years as these children and adolescents enter adulthood.

\section{References}

1. Taylor AJ, Rogan KM, Virmani R. Sudden cardiac death associated with isolated congenital coronary artery anomalies. J Am Coll Cardiol. 1992;20:640-7.

2. Basso C, Maron BJ, Corrado D, Thiene G. Clinical profile of congenital coronary artery anomalies with origin from the wrong aortic sinus leading to sudden death in young competitive athletes. J Am Coll Cardiol. 2000;35:1493-501.

3. Cheitlin MD, DeCastro CM, McAllister HA. Sudden death as a complication of anomalous left coronary origin from the anterior sinus of Valsalva: a not-so-minor congenital anomaly. Circulation. 1974;50:780-7.

4. Barth CW III, Roberts WC. Left main coronary artery originating from the right sinus of Valsalva and coursing between the aorta and pulmonary trunk. J Am Coll Cardiol. 1986;7:366-73.

5. Frescura C, Basso C, Thiene G, et al. Anomalous origin of coronary arteries and risk of sudden death: a study based on an autopsy population of congenital heart disease. Hum Pathol. 1998;29:689-95.

6. Kragel AH, Roberts WC. Anomalous origin of either the right or left main coronary artery from the aorta with subsequent coursing between aorta and pulmonary trunk: analysis of 32 necropsy cases. Am J Cardiol. 1988;62:771-7.

7. Burke AP, Farb A, Virmani R, Goodin J, Smialek JE. Sports-related and nonsports-related sudden cardiac death in young adults. Am Heart J. 1991;121: 568-75.

8. Corrado D, Thiene G, Nava A, Rossi L, Pennelli N. Sudden death in young competitive athletes: clinicopathologic correlations in 22 cases. Am J Med. 1990;89: 588-96.

9. Brothers JA, McBride MG, Seliem MA, Marino BM, Tomlinson RS, Pampaloni MH, et al. Evaluation of myocardial ischemia following surgical repair of anomalous aortic origin of a coronary artery from the opposite sinus of Valsalva in a series of pediatric patients. $J$ Am Coll Cardiol. 2007;50:2078-82.

10. Romp RL, Herlong JR, Landolfo CK, et al. Outcome of unroofing procedure for repair of anomalous aortic origin of left or right coronary artery. Ann Thorac Surg. 2003;76:589-96.

11. Graham TP Jr, Driscoll DJ, Gersony WM, Newburger JW, Rocchini A, Towbin JA. 36th Bethesda conference: eligibility recommendations for competitive athletes with cardiovascular abnormalities. Task Force 2: Congenital Heart Disease. J Am Coll Cardiol. 2005;45:1326-33.

12. Beaver WL, Wasserman K, Whipp BJ. A new method for detecting anaerobic threshold by gas exchange. J Appl Physiol. 1986;60:2020-7.

13. Cooper DM, Weiler-Ravell D, Whipp BJ, Wasserman K. Aerobic parameters of exercise as a function of body size during growth in children. $J$ Appl Physiol. 1984;56:628-34

14. Kuczmarski RJ, Ogden CL, Grummer-Strawn LM, Flegal KM, Guo SS, Wei R, et al. CDC growth charts: United States. Advance data from vital and health statistics; no. 314. Hyattsville, Maryland:: National Center for Health Statistics; 2000. 
15. Varni JW, Seid M, Rode CA. The PedsQL 4.0: Measurement model for the Pediatric Quality of Life Inventory. Med Care. 1999;37:126-39.

16. Varni JW, Seid M, Kurtin PS. The PedsQL 4.0: reliability and validity of the Pediatric Quality of Life Inventory version 4.0 generic core scales in healthy and patient populations. Med Care. 2001;39:800-12.

17. Varni JW, Seid M, Knight TS, Uzark K, Szer IS. The PedsQL 4.0 Generic Core Scales: sensitivity, responsiveness, and impact on clinical decision-making. J Behav Med. 2002;25:175-93.
18. Varni JW, Burwinkle TM, Seid M, Skarr D. The PedsQL 4.0 as a pediatric population health measure: feasibility, reliability, and validity. Ambul Pediatr. 2003; 3:329-41.

19. Fricker FJ, Trento A, Griffith BP. Pediatric cardiac transplantation. Cardiovasc Clin. 1990;20:223-35.

20. Hirshfeld AB, Kahle AL, Clark BJ, Bridges ND. Parent-reported health status after pediatric thoracic organ transplant. J Heart Lung Transplant. 2004;23: 1111-8. 